\title{
Using heat to scan transparent surfaces
}

Gonen Eren, Olivier Aubreton, Fabrice Meriaudeau, L. A. Sanchez Secades, David Fofi, Frederic Truchetet, and Aytul Ercil

A novel approach to $3 D$ scanning holds promise for imaging seethrough objects, such as those made from glass.

Today, with quality control gaining importance, reconstruction solutions that lead to a full-quality 2D and 3D inspection are being developed. However, when objects are highly transparent or highly specular, 3D reconstruction becomes very difficult. Classical scanning approaches using light patterns projected on the surface and triangulation are neither efficient nor reliable for transparent materials. For instance, a glass bottle scanned by a Minolta VI-910 Non Contact 3D Digitizer cannot be properly reconstructed because of reflections and refractions of the projected laser line.

Researchers have proposed different approaches for handling transparent objects, including the shape-from-distortion technique to recover the surface shape of a glass object, ${ }^{1}$ and the structure-from-motion ${ }^{2,3}$ technique. Others ${ }^{4}$ investigated several applications of direct ray measurement and proposed a practical algorithm to reconstruct two interface refractive light interactions using a three-viewpoint setup and measuring the exitant ray directions. Lastly, several studies have proposed using shape-form polarization. 5, 6

The approach presented here, called 'scanning from heating ${ }^{7}$ (SFH), benefits from two interesting properties of glass material: its opacity in the IR domain and its emissivity. Figure 1 illustrates the percentage of light transmission in the IR domain of the most commonly used glasses. For each type, there is a wavelength such that transmission is close to $0 \%$. This wavelength is called the IR cut-off, and it depends on the glass composition. ${ }^{8}$ At a wavelength higher than $10 \mu \mathrm{m}$, the glasses presented in Figure 2 can be considered as opaque. Figure 1(b) shows an image of a silicon dioxide glass bottle placed in front of an IR illumination and observed with a long-wave IR camera sensitive to $8-13 \mu \mathrm{m}$. The object does not transmit the light coming from the source and appears to be opaque.

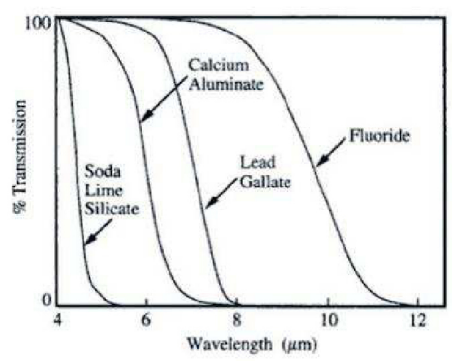

a)

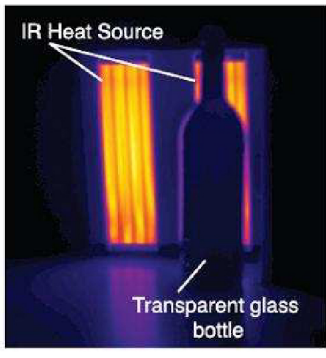

b)
Figure 1. a) Percentage of light transmission in the IR domain of commonly used glasses b) Image taken with a long wave IR camera sensitive to $8-13 \mu \mathrm{m}$.

The second property of glass material is its emissivity. All substances continuously emit electromagnetic radiation because of the molecular and atomic agitation associated with their internal energy, which is proportional to the material temperature. Emissivity specifies how well a real object radiates energy compared to an ideal body, called a blackbody. (A blackbody is considered a perfect absorber and emitter in each direction at every wavelength.) For glass, which has the angular emissivity of a dielectric sphere, the radiation approaches a Lambertian source.

The choice of a wavelength range up to $10 \mu \mathrm{m}$ makes it possible to overcome the problem of transparency. However, the key step is to locally heat the surface and then observe the radiation emitted by it.

The idea is shown in Figure 2. $P_{1}$ is the heated point at the surface of the object at Position 1 , and $\left(X_{1}, Y_{1}, Z_{1}\right)$ are its world coordinates. The projection of $P_{1}$ on the camera plane is called $P_{c 1}$, and its coordinates $\left(X_{c 1}, Y_{c 1}\right)$ are in the camera coordinate system $\left(O_{c}, X_{c}, Y_{c}\right) . P_{2}$ is the heated point at Position 2 , and $\left(X_{2}, Y_{2}, Z_{2}\right)$ are its world coordinates. The projection of $P_{2}$ on the camera plane is called $P_{c 2}$, and its coordinates are $\left(X_{c 2}, Y_{c 2}\right)$. The variation of height between $P_{1}$ and $P_{2}$, called $Z$, can be obtained by triangulation using a pinhole camera model. 


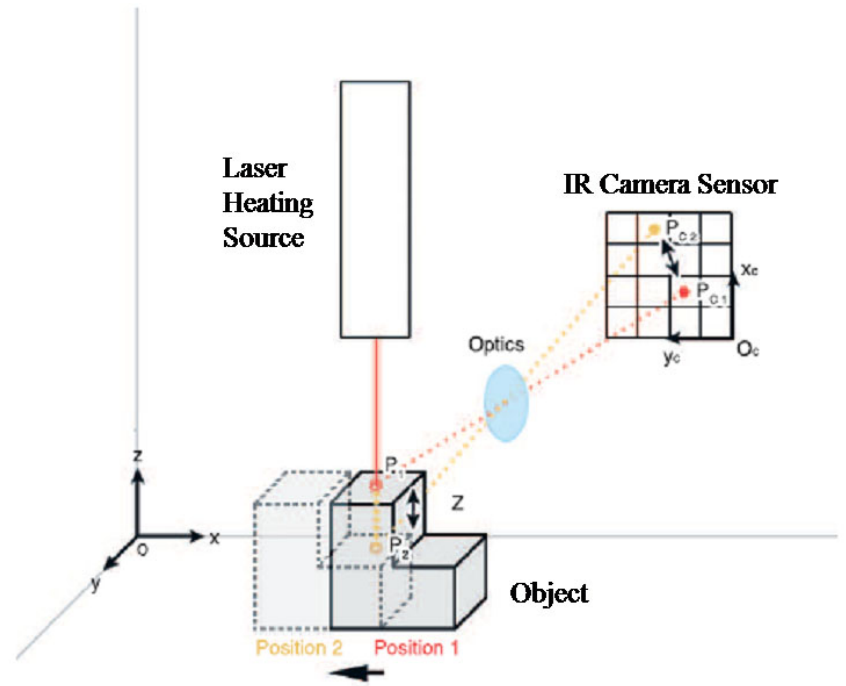

Moving Direction of the Object

Figure 2. A schematic of the method. $P_{1}$ and $P_{2}$ are the heated points at the surface of the object at Positions 1 and 2, respectively, and $Z$ is the height variation between the two points. $P_{c 1}$ and $P_{c 2}$ are the projection of $P_{1}$ and $P_{2}$ on the camera plane, respectively.



a)

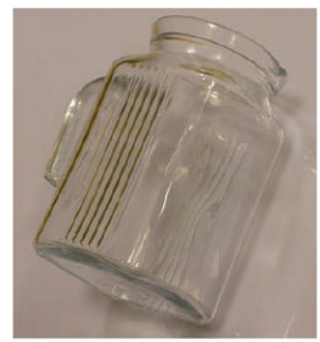

c)

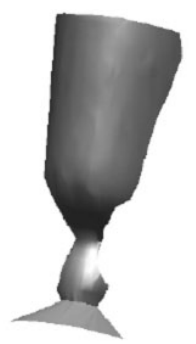

b)

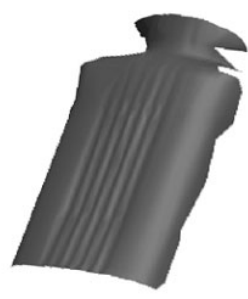

d)
Figure 3. Transparent objects $a$ ), c) and their $3 D$ reconstructions $b$ ), $d$ ) using the SFH method.

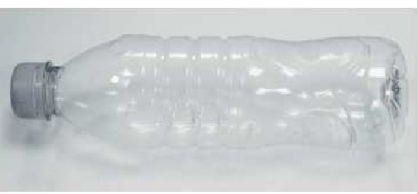

(a)

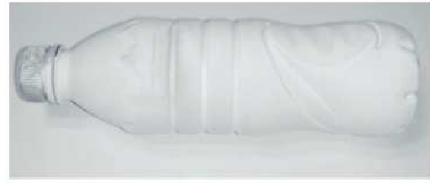

(c)

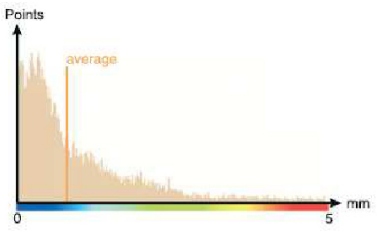



(b)

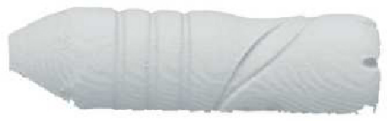

(d)

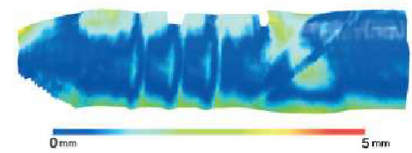

Figure 4. (a) A transparent plastic bottle was (b) reconstructed using the SFH method. (c) A powdered plastic bottle was (d) reconstructed with a Minolta VI-910 Non Contact 3D Digitizer. (e) Histogram and (f) $3 D$ representation of the difference between the two reconstructions.

Figure 3 shows the results from scanning a complex transparent glass object $7 \times 7 \times 15 \mathrm{~cm}$ in size from 2100 points and results obtained from another complex transparent glass object $9 \times 9 \times 14 \mathrm{~cm}$ in size from 1300 points. This demonstrates that the system can be applied to a wide range of objects-from surfaces used in the automotive industry to more complex objects in the packaging industry.

In this experimental setup, we used a $\mathrm{CO}_{2}$ laser, but the same principle can be applied with other heating systems so that the material absorbs the incident radiation and emits radiations in the IR domain. Figure 4 compares a 3D reconstruction of a plastic bottle by the SFH method to one obtained by a Minolta VI-910 Non Contact 3D Digitizer of the same bottle, powdered on the surface. The results fit reasonably well, and the average deviation is $200 \mu \mathrm{m}$.

In summary, we developed a new method to determine the surface shape of transparent objects using laser surface heating and thermal imaging. The results for glass objects are promising. Future work will include the design of an experimental system that can project a specific pattern (line, grid, matrix of points, etc.) to improve the $3 \mathrm{D}$ point acquisition speed. While we have only studied the method for transparent glass, we will also study its application to different materials (and the effects on the reconstruction, laser type, and power). Finally, we envisage an industrial prototype. 


\section{Author Information}

\section{Gonen Eren}

Computer Vision and Pattern Analysis (VPA) Laboratory

Sabanci University

Istanbul, Turkey

http://http://vpa.sabanciuniv.edu

Laboratory of Electronics, Computer Science, and Image Processing (Le2i)

University of Burgundy

Le Creusot, France

http://http://www.Le2i.com

Gonen Eren received his master's in computer engineering in 2007 from Galatasaray University, in Istanbul, Turkey. He is currently enrolled in a double $\mathrm{PhD}$ program.

Olivier Aubreton, Fabrice Meriaudeau , L. A. Sanchez Secades, David Fofi , and Frederic Truchetet

Le2i

University of Burgundy

Le Creusot, France

\section{Aytul Ercil}

VPA Laboratory

Sabanci University

Istanbul, Turkey

\section{References}

1. S. Hata, Y. Saitoh, S. Kumamura, and K. Kaida, Shape extraction of transparent object using genetic algorithm, Proc. 13th Int'1 Conf. Pattern Recogn., pp. 684-688, 1996.

2. M. Ben-Ezra and S. Nayar, What does motion reveal about transparency?, 9th IEEE Int'l Conf. Comput. Vision 2, pp. 1025-1032, 2003.

3. S. Agarwal, S. P. Mallick, D. Kriegman, and S. Belongie, On refractive optical flow, 8th Euro. Conf. Comput. Vision, pp. 483-494, 2004

4. K. Kutulakos and E. Steger, A theory of refractive and specular 3D shape by light-path triangulation, 10th IEEE Int'1 Conf. Comput. Vision 2, pp. 1448-1455, 2005.

5. D. Miyazaki and K. Ikeuchi, Inverse polarization raytracing: estimating surface shapes of transparent objects, Proc. 2005 IEEE Conf. Comput. Vision and Pattern Recogn. 2, pp. 910-917, 2005.

6. D. Miyazaki, M. Saito, Y. Sato, and K. Ikeuchi, Determining surface orientations of transparent objects based on polarization degrees in visible and infrared wavelengths, J. Opt. Soc. Am. A 19, pp. 687-694, 2002.

7. G. Eren, O. Aubreton, F. Meriaudeau, L. A. Sanchez Secades, D. Fofi1, A. T. Naskali, F. Truchetet, and A. Ercil, Scanning From Heating: 3D shape estimation of transparent objects from local surface heating, Opt. Express 17 (14), pp. 11457-11468, 2009.

8. J. E. Shelby, Introduction to Glass Science and Technology, Royal Society of Chemistry, Cambridge, 2005. doi:10.1039/9781847551160 